\title{
Near-Normal Incidence Dark-Field Microscopy: Applications to Nanoplasmonic Spectroscopy
}

\author{
Jonathan A. Fan, ${ }^{* \dagger, \ddagger}$ Kui Bao, ${ }^{\S}$ J. Britt Lassiter, ${ }^{\S}$ Jiming Bao, ${ }^{\prime \prime}$ Naomi J. Halas, ${ }^{\S, \perp}$ Peter Nordlander, ${ }^{\S}$ \\ and Federico Capasso \\ ${ }^{\dagger}$ Beckman Institute, University of Illinois, 405 North Mathews Avenue, Urbana, Illinois 61801, United States \\ ${ }^{\ddagger}$ School of Engineering and Applied Sciences, Harvard University, 9 Oxford St., Cambridge, Massachusetts 02138, United States \\ ${ }^{\S}$ Department of Physics, Rice University, 6100 Main St., Houston, Texas 77005, United States \\ "Department of Electrical and Computer Engineering, University of Houston, 4800 Calhoun Road, Houston, Texas 77204, United \\ States \\ ${ }^{\perp}$ Department of Chemistry, Rice University, 6100 Main St., Houston, Texas 77005, United States
}

\section{Supporting Information}

\begin{abstract}
The spectroscopic characterization of individual nanostructures is of fundamental importance to understanding a broad range of physical and chemical processes. One general and powerful technique that addresses this aim is dark-field microscopy, with which the scattered light from an individual structure can be analyzed with minimal background noise. We present the spectroscopic analysis of individual plasmonic nanostructures using dark-field illumination with incidence nearly normal to the substrate. We show that, compared to large incidence angle approaches, the near-normal incidence approach
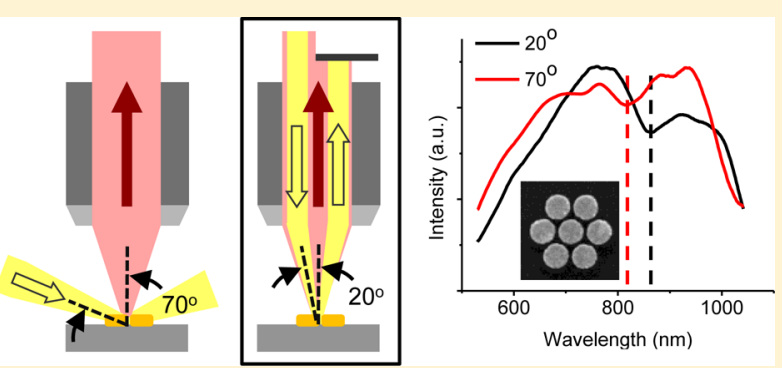
provides significantly higher signal-to-background ratios and reduced retardation field effects. To demonstrate the utility of this technique, we characterize an individual chemically synthesized gold nanoshell and a lithographically defined heptamer exhibiting a pronounced Fano-like resonance. We show that the line shape of the latter strongly depends on the incidence angle. Nearnormal incidence dark-field microscopy can be used to characterize a broad range of molecules and nanostructures and can be adapted to most microscopy setups.
\end{abstract}

KEYWORDS: Dark field, nanoplasmonics, spectroscopy, polarization, Fano resonance, nanoshell

$\mathrm{P}$ lasmonic nanostructures are the topic of a broad range of fundamental and applied research because their optical properties strongly depend on their geometry, material composition, and local dielectric environment. As such, they can be engineered to yield electric, ${ }^{1}$ magnetic, ${ }^{2}$ and Fano resonances 3,4 across the visible spectrum, thereby forming a basis for designer optical materials. Applications include various imaging and detection schemes. One example is localized surface plasmon resonance sensing, in which the local presence of a liquid, ${ }^{5}$ biological material, ${ }^{6}$ or gas $^{7}$ can be detected via shifts in the plasmon resonance spectrum. In another example, ensembles of particles with different geometries can be used as multiplexed optical barcodes in biological labeling applications. ${ }^{8,9}$ Coupled plasmonic structures have uses in various field-enhanced spectroscopies such as surface-enhanced Raman spectroscopy, where the detection and fingerprinting of localized molecules are possible with a single nanostructure. ${ }^{10}$ For many of these applications, it is essential to characterize individual nanostructures; in this limit, detection schemes can operate with nanoscale spatial resolution, the uniqueness of different nanostructures in an ensemble can be exploited, and bulk effects such as inhomogeneous broadening, orientation averaging of anisotropic particles, and array effects can be eliminated.

Dark-field microscopy is a widely utilized tool for detecting and characterizing nanostructures. ${ }^{8,11-13}$ In this technique, an incident beam illuminates the sample of interest, and scattered radiation from the nanoscale objects is collected by a microscope objective and analyzed in a spectrometer. The reflection of the incident beam from the substrate is not collected by the spectrometer to minimize background noise. A pinhole at the spectrometer entrance can be used in a confocallike design to ensure that scattered light from only a single nanostructure enters the spectrometer. Typical commercial setups employ dark-field objectives, which combine the collection objective with the dark-field condenser. Here, the incidence angle of the illumination beam is set to be larger than the angle defined by the numerical aperture of the collection objective. The beam is defocused, such that the illuminated area spans the field of view (see Supporting Information). While this

Received: January 13, 2012

Revised: April 17, 2012

Published: April 23, 2012 


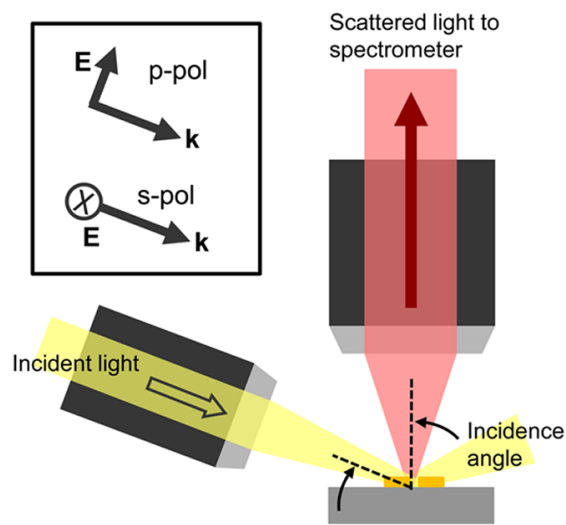

b

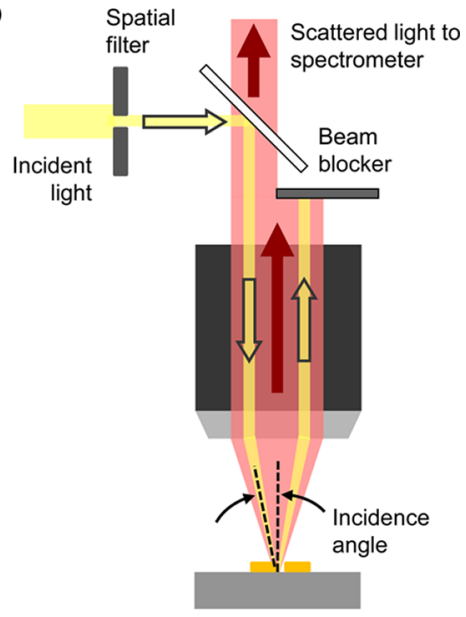

Figure 1. Schematics of two different dark-field spectroscopy configurations. (a) Dark-field setup with a large angle of incidence. One objective is used to lightly focus polarized white light on the substrate while a second objective collects the scattered light. The inset indicates the electric field orientations for s- and p-polarized incident light. (b) Dark-field setup with a small angle of incidence. Here, a single objective is used to both focus white light on the sample and collect the scattered light. The incident beam is collimated, spatially filtered, and polarized before it is directed parallel to the objective by a beam splitter. A beam blocker is used to prevent the reflected white light beam from entering the spectrometer.

technique is effective for many dark-field imaging applications, it is inflexible in many ways: the incidence angle, the numerical aperture of the collection objective, and the polarization of incident light are fixed. It would be of general interest to develop new dark-field microscopy techniques that circumvent the need for specialized dark-field objectives and that provide the means for new optical analyses.

In this study, we probe the optical properties of individual plasmonic nanostructures using a dark-field illumination scheme with near-normal incidence. This technique is compared with a dark-field illumination scheme with large incidence angle, which is more typical of standard dark-field techniques. We show that the near-normal incidence approach offers clear advantages over the large incidence angle approach such as significantly higher signal-to-background ratio and reduced retardation field effects. Schematics of both techniques are presented in Figure 1, and they clearly employ dark-field illumination because in both cases, the incident light beam reflected from the substrate is not collected in the spectrometer. Both techniques utilize ordinary microscope objectives to collect the scattered light. The large incidence angle technique has been used previously for single nanostructure studies, ${ }^{4}$ and sample illumination is achieved by polarizing and lightly focusing radiation onto the substrate with a condenser objective (Figure 1a).

The near-normal incidence technique is one that has been previously used in applications such as particle trapping, but to the best of our knowledge, it has not been used before for nanoplasmonic spectroscopy. We also note the existence of a complementary technique, dark-field forward scattering microscopy, which is suitable for systems utilizing transparent and planar substrates. ${ }^{14}$ In our scheme, the incident light is collimated, polarized, spatially filtered, and aligned parallel with the collection objective in a manner detailed in Figure $1 \mathrm{~b}$. The spatial filter here controls the size and shape of the incident beam and its incidence position on the back facet of the objective lens. Upon focusing, this light illuminates the sample with approximately s-polarization. A beam blocker is used to prevent incident light reflected from the sample substrate from entering the spectrometer, albeit at the expense of blocking some collected scattered radiation from entering the spectrometer. As the collection objective also functions as the condenser here, the incidence angle has an upper limit set by the numerical aperture of the objective; this angle can be tuned by changing the distance of the incident beam relative to the axis of the objective. In the following experiments, we use a collection objective with $50 \times$ magnification and a numerical aperture of 0.65 , and our incidence angles for near-normal and large angle illumination are set to $20^{\circ}$ and $70^{\circ}$, respectively.

To demonstrate the utility of our new approach, we first investigate the optical properties of an individual gold nanoshell on a $\mathrm{ZnSe}$ substrate (Figure 2). Nanoshells are chemically synthesized silica-core gold-shell nanostructures, and their resonances can be tuned across the visible and infrared spectrum by varying their core-shell geometry. ${ }^{15}$ When placed in a background of uniform dielectric constant, they have polarization-independent optical responses; in other words, their dipolar and quadrupolar modes are degenerate. However, their optical responses become highly polarization dependent when the particles are deposited on a high refractive index substrate $\left(n_{\mathrm{ZnSe}}=2.67\right.$ at $\left.550 \mathrm{~nm}\right)$ due to the interaction between their plasmonic modes and corresponding substrate image charges. ${ }^{16-18}$ As such, the formerly degenerate dipolar and quadrupolar modes each split into new and different orientation-dependent modes; furthermore, the presence of the substrate can mediate the coupling and hybridization of these dipolar and quadrupolar modes. A detailed discussion of these modes and their coupling can be found in ref 17 .

The s- and p-polarized nanoshell spectra are distinctly different when excited at a large incidence angle (Figure 2a). The s-polarized spectrum contains a broad electric dipole peaked near $700 \mathrm{~nm}$ and a narrow electric quadrupole peaked near $600 \mathrm{~nm}$. The quadrupole mode is optically active and clearly visible here because of the hybridization of the dipolar (symmetry $D_{2}$ ) and quadrupolar (symmetry $Q_{1}$ ) modes ${ }^{17,18}$ (see bottom inset). This hybridization is mediated by the substrate, which supports image charges that are similar for both of these modes (see bottom inset, red). The p-polarized spectrum does not contain such a pronounced quadrupole mode; however, its electric dipole mode is substantially 

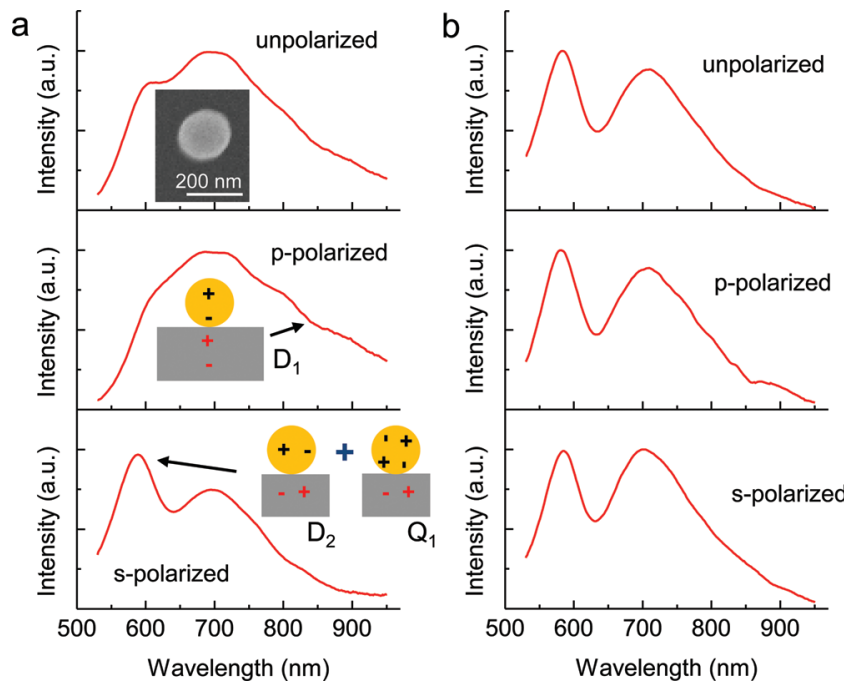

Figure 2. Spectra of an individual nanoshell on a $\mathrm{ZnSe}$ substrate. (a) TEM image (top inset) and spectra of a nanoshell for a white light incidence angle of $70^{\circ}$. The nanoshell has an inner silica core diameter of $125 \mathrm{~nm}$ and a gold shell thickness of $30 \mathrm{~nm}$. The s- and p-polarized spectra differ due to the highly polarization-dependent interaction between the particle and substrate. The p-polarized spectrum is denoted by a broad electric dipole peak of $D_{1}$ symmetry that arises from longitudinal coupling between the nanoshell plasmon mode (dark $+/-$ ) and image charges (red $+/-$ ) in the substrate (middle inset). The s-polarized spectrum is characterized by a distinct and narrow peak near $600 \mathrm{~nm}$, which is an optically active quadrupole mode that is visible due to substrate-induced quadrupole mode hybridization with the dipole plasmon mode (bottom inset). (b) Spectra of the same nanoshell for a white light incidence angle of $\sim 20^{\circ}$. For all polarizations, the spectra are characterized by a strong quadrupolar peak near $600 \mathrm{~nm}$, similar to that in the s-polarized spectrum in (a).

broadened. This effect is due to strong capacitive coupling of the particle dipole mode with its image charges in the substrate $^{17}$ (see middle inset). The unpolarized spectrum is a superposition of the s- and p-polarized spectra and contains signatures of both the s-polarized quadrupole mode and broadened p-polarized dipole mode.

As the incidence angle is reduced to the near-normal configuration, the s-polarized, p-polarized, and unpolarized spectra of the same nanoshell exhibit comparable line shapes that each contain a strong electric quadrupole mode near 600 $\mathrm{nm}$ (Figure $2 \mathrm{~b}$ ), similar to the s-polarized spectrum from Figure 2a. This polarization independence and the presence of the quadrupolar peak indicate that for all incident polarization configurations, this illumination technique principally excites the lateral modes of the nanoshell. This is consistent with our expectation of near-normal incidence excitation, where the electric field of the incident radiation is always parallel or nearly parallel to the substrate surface.

We note that the signal-to-background ratio of the nearnormal measurements at visible frequencies is approximately 8:1 while the signal-to-background ratio of the large incidence angle s-polarized measurements is only approximately 2:1 (see Supporting Information for spectra). Much of this collected background appears to be due to random multiple scattering from the substrate. One of the reasons why the near-normal incidence technique yields an enhancement in signal-tobackground is because in this scheme, the incident light gets better focused on the substrate: with the lenses and fiber optics used here, the illumination spot size is $\sim 20 \mu \mathrm{m}$ in diameter (see Supporting Information for discussion). On the other hand, the large incidence angle scheme illuminates a spot size that is on the order of square millimeters; this spot is larger because beams with large incidence angles project over large areas on a substrate and also because it does not use the $50 \times$ objective to further focus the spot. With the near-normal incidence scheme, the spatial extent of substrate illumination and subsequent extent of background created by multiple scattering events can be limited.

We also show that these two dark-field illumination techniques can be used together to uniquely probe the optical properties of more complex nanostructures. As a model system, lithographically defined gold heptamer clusters are studied. Heptamers are of general interest because they support Fanolike resonances, which arise from interference between subradiant "dark" modes and superradiant "bright" modes and which are characterized by narrow minima in their scattering spectra. ${ }^{3,4,19-25}$ The heptamers here are fabricated by electron beam lithography: the nanostructure pattern is first defined in a layer of PMMA, metal is deposited $(2 \mathrm{~nm} \mathrm{Ti} / 30$ $\mathrm{nm} \mathrm{Au}$ ), and the excess metal is finally lifted off. The final nanostructures consist of $150 \mathrm{~nm}$ diameter disks separated by $15 \mathrm{~nm}$ gaps. The individual heptamers are spaced $50 \mu \mathrm{m}$ apart to ensure the ease of single nanostructure spectroscopy. The substrate is silicon with a $100 \mathrm{~nm}$ thick thermally grown oxide layer; here, the oxide layer is sufficiently thick as to minimize coupling between the heptamer plasmon modes and their corresponding image charges in the high refractive index silicon substrate. $^{17}$ A scanning electron microscope image of an individual heptamer is presented in the inset of Figure $3 \mathrm{a}$.

It is noted though that, due to reflections of scattered light at the silicon-silica interface, the detected signal comes from multiple sources. The principal source is radiation that scatters from the heptamer and goes directly into the detector. There is also radiation that scatters into the substrate and gets reflected from the silicon-silica interface. Some of this radiation goes directly into the detector while the rest is rescattered by the heptamer, both in the direction of the detector and in the direction of the silicon-silica interface (which leads to additional rescattering events). As such, the detected spectrum is actually a combination of interfering sources. The effect of this interference can be judged from spectra of the heptamer with and without a silicon-silica interface (see the Supporting Information). We see that with the silicon-silica interface, small peaks in the spectra due to this interference become clearly visible. We also note that this interference does not strongly affect the Fano minima, as their positions do not get strongly modified.

The s-polarized spectra of an individual heptamer are measured with both illumination techniques and are plotted in Figure 3a. We see that both spectra here exhibit clear Fano resonances, with local intensity dips delineated by dashed lines. Theoretical spectra are calculated using the finite-element analysis program COMSOL, where the heptamer geometry, silica-silicon substrate, and light collection geometries match those of the experiment. These spectra generally match well with theory (Figure 3b); however, the magnitudes of the Fano dips are smaller in the experimental spectra than in the theoretical ones, which is likely due to fabrication errors, the quality of deposited metal, and the absence of the $2 \mathrm{~nm}$ thick titanium adhesion layer in the simulations. 

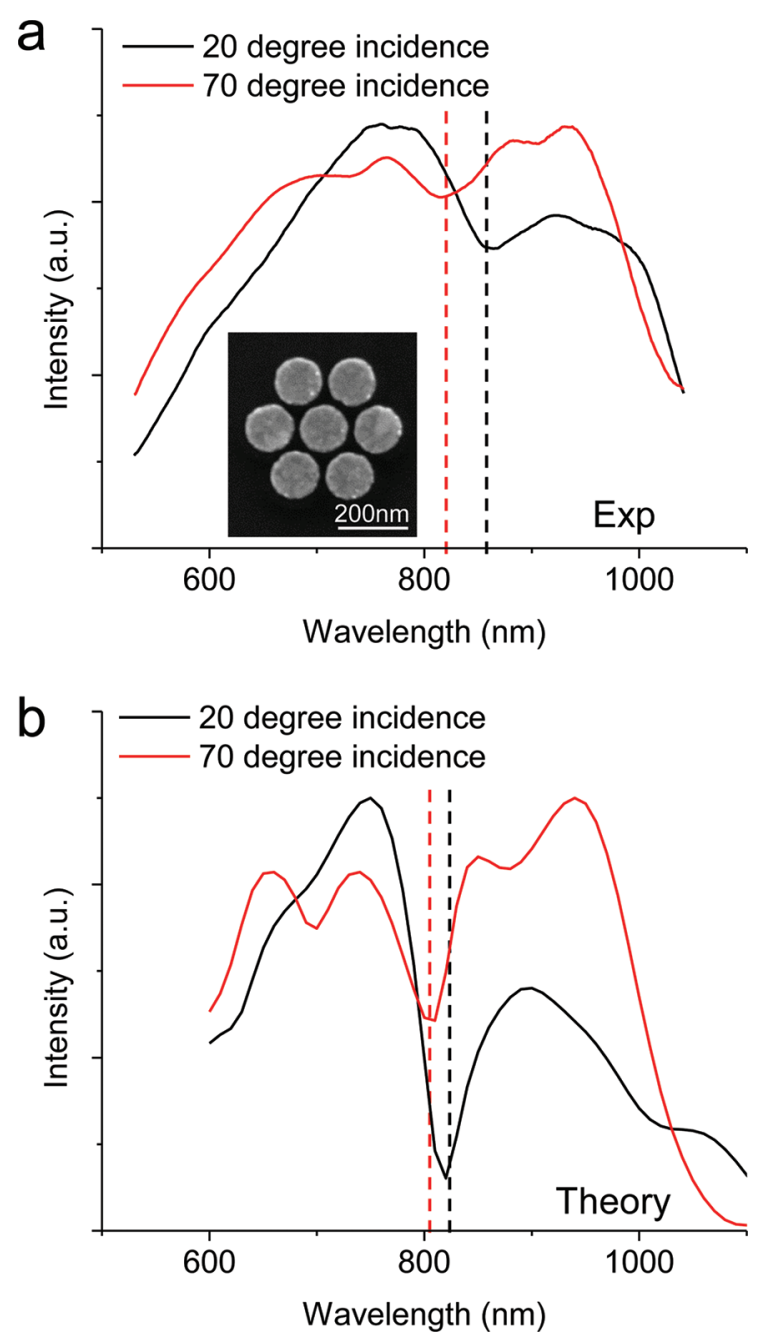

Figure 3. Spectra of an individual lithographically defined plasmonic heptamer. (a) Experimental s-polarized spectra of a heptamer measured for two different incident angles of illumination. The Fano minima are denoted by dashed lines. The inset shows an SEM image of the nanostructure; the average disk diameter and interparticle gap distance are 144 and $15 \mathrm{~nm}$, respectively. (b) Theoretical scattering spectra of the same structure above, where the sample geometry, illumination angle, and radiation collection geometry are based on experimental parameters. As in the experimental spectra above, these spectra exhibit a change of the Fano minimum line shape as a function of incidence angle.

We point out a few discernible differences between these two experimental spectra. One is that the large incidence angle spectrum contains a series of small peaks that are absent in the near-normal incidence spectrum. Some of these peaks arise from the direct coupling of the incident field to these dark modes, which is due to retardation effects. ${ }^{26}$ The excitation of dark modes with this mechanism has been previously studied in other plasmonic structures such as rings, ${ }^{27}$ where dark quadrupole modes were excited with large incidence angle illumination. This occurs because as the angle of incidence becomes large, the electric field across the nanostructure is no longer uniform and, as such, can directly couple to multipolar plasmon modes. The "peak" near $950 \mathrm{~nm}$ includes a magnetic dipole peak superimposed on the broad electric dipole peak of the cluster. This mode arises due to the large magnetic field component of the incident wave pointing out of the heptamer plane, which induces a circulating current.
One other difference between these spectra is that the Fano minimum in the large incidence angle spectrum has a smaller magnitude (relative to the peak near $800 \mathrm{~nm}$ ) and is blueshifted relative to that in the near-normal incidence spectrum. This is peculiar because the bright and dark plasmonic modes causing Fano interference are the same in both cases; the change of incidence angle must be modifying the interference between these modes. The explanation that we propose is that at large incidence angles, incident radiation directly couples into the dark mode by retardation, similar to its coupling into other heptamer dark modes as discussed earlier. As such, this new excitation condition modifies the relative phase of the dark and bright modes and therefore the Fano line shape.

To further elucidate these effects, it is useful to recall that the Fano minimum appears at the frequency $f_{0}$ when the total phase shift from the energy transfer process $|I\rangle \rightarrow|B\rangle \rightarrow|D\rangle \rightarrow$ $|B\rangle$ is $180^{\circ}$ out of phase with $|I\rangle \rightarrow|B\rangle$, where $|I\rangle$ is the incident white light source and $|B\rangle$ and $|D\rangle$ represent the bright and dark mode states, respectively. ${ }^{21}$ Phase shifts arise because these modes, which can be modeled as classical oscillators, ${ }^{28,29}$ couple with each other near their resonances, thereby incurring a frequency-dependent phase response during each coupling event (i.e., $|B\rangle \rightarrow|D\rangle$ and $|D\rangle \rightarrow|B\rangle$ ). When retardation is introduced, the Fano minimum condition is modified and occurs when the pathways $|I\rangle \rightarrow|B\rangle$ and $|I\rangle \rightarrow|B\rangle \stackrel{I_{\text {Ret }}}{\longrightarrow}|D\rangle \rightarrow$ $|B\rangle$ are $180^{\circ}$ out of phase. $I_{\text {Ret }}$ represents the incident retarded field excitation. The dark mode now gets excited by both the bright mode and the incident retarded field, which together yield a combined phase response at $\mathrm{f}_{0}$ that is phase advanced compared to before (with no $I_{\text {Ret }}$ ). Now, to satisfy the Fano minimum condition, this phase-advanced dark mode response must be compensated; this is accomplished by exciting the dark mode at frequencies blue-shifted from $f_{0}$, because phase response (i.e., phase delay) increases with frequency in a classical oscillator. The result is a blue-shift of the Fano dip. It is noted that, within the frequency range of the narrow-band dark mode, the phase response of $|D\rangle \rightarrow|B\rangle$ is effectively fixed and does not change with the introduction of $I_{\text {Ret }}$ due to the broad line width of the bright mode.

In conclusion, we have presented the dark-field spectroscopy of individual plasmonic nanostructures using nearly normal incidence. Together with more conventional large-angle incidence dark-field microscopy, it is possible to realize the dark-field illumination of a nanostructure with nearly arbitrary incidence angle. Large-angle and near-normal illumination each yield particular traits. Large-angle illumination can be used to probe the dark modes of nanostructures, as excited by retardation, and be used to excite longitudinal modes and magnetic modes with p-polarized incidence light. With nearnormal incidence, retardation effects are minimized, and there exists the potential for enhanced signal-to-background. This technique also eliminates any excitation of modes perpendicular to the substrate, so that no polarizer is required for the characterization of structures with in-plane symmetry. Both techniques can be used together to perform new types of optical analyses, and in this study, Fano resonances are probed in gold heptamers. In the context of Fano structures, it would be of interest to probe systems of different geometries, sizes, and symmetries ${ }^{20,30}$ with these techniques in future study. While plasmonic systems are examined here, these techniques are general to analyzing scattered radiation from any nanoscale source. We believe that these techniques will have broad 
implications in scattering spectroscopy, and their ease of setup and use of basic microscopy components will make them readily adaptable to most microscopy systems and setups.

\section{ASSOCIATED CONTENT}

\section{S Supporting Information}

Details pertaining to the experimental setup, signal-to-background analysis, and substrate effects. This material is available free of charge via the Internet at http://pubs.acs.org.

\section{AUTHOR INFORMATION}

\section{Corresponding Author}

*E-mail: jonfan@illinois.edu.

\section{Notes}

The authors declare no competing financial interest.

\section{ACKNOWLEDGMENTS}

J.A.F. and F.C. acknowledge the NSF Nanoscale Science and Engineering Center (NSEC). J.A.F. thanks Q. Zhang and X. Lu for experimental help. P.N., N.H., K.B., and J.B.L. acknowledge support from the Robert A. Welch foundation (C-1222 and C1220), the National Science Foundation (ECCS-1040478), the US Department of Defense NSEFF program (N00244-09-10067), the Defense Threat Reduction Agency (DTRA) HDTRA1-11-1-0040, and the Office of Naval Research (N00244-09-1-0989). J.M.B. acknowledges support from the Robert A. Welch Foundation (E-1728).

\section{REFERENCES}

(1) Kelly, K. L.; Coronado, E.; Zhao, L. L.; Schatz, G. C. The optical properties of metal nanoparticles: The influence of size, shape, and dielectric environment. J. Phys. Chem. B 2003, 107, 668-677.

(2) Cai, W. S.; et al. Metamagnetics with rainbow colors. Opt. Express 2007, 15, 3333-3341.

(3) Luk'yanchuk, B.; et al. The Fano resonance in plasmonic nanostructures and metamaterials. Nat. Mater. 2010, 9, 707-715, DOI: $10.1038 /$ nmat2810.

(4) Fan, J. A.; et al. Self-Assembled Plasmonic Nanoparticle Clusters. Science 2010, 328, 1135-1138.

(5) Mock, J. J.; Smith, D. R; Schultz, S. Local refractive index dependence of plasmon resonance spectra from individual nanoparticles. Nano Lett. 2003, 3, 485-491.

(6) Anker, J. N.; et al. Biosensing with plasmonic nanosensors. Nat. Mater. 2008, 7, 442-453.

(7) Liu, N.; Tang, M. L.; Hentschel, M.; Giessen, H.; Alivisatos, A. P. Nanoantenna-enhanced gas sensing in a single tailored nanofocus. Nat. Mater. 2011, 10, 631-636.

(8) Mock, J. J.; Barbic, M.; Smith, D. R.; Schultz, D. A.; Schultz, S. Shape effects in plasmon resonance of individual colloidal silver nanoparticles. J. Chem. Phys. 2002, 116, 6755-6759.

(9) Nicewarner-Peña, S. R.; et al. Submicrometer Metallic Barcodes. Science 2001, 294, 137-141.

(10) Xu, H. X.; Bjerneld, E. J.; Kall, M.; Borjesson, L. Spectroscopy of single hemoglobin molecules by surface enhanced Raman scattering. Phys. Rev. Lett. 1999, 83, 4357-4360.

(11) Hu, M.; et al. Dark-field microscopy studies of single metal nanoparticles: understanding the factors that influence the linewidth of the localized surface plasmon resonance. J. Mater. Chem. 2008, 18, 1949-1960.

(12) Nehl, C. L.; et al. Scattering Spectra of Single Gold Nanoshells. Nano Lett. 2004, 4, 2355-2359.

(13) Knight, M. W.; Fan, J.; Capasso, F.; Halas, N. J. Influence of excitation and collection geometry on the dark field spectra of individual plasmonic nanostructures. Opt. Express 2010, 18, 25792587.
(14) Thar, R.; Blackburn, N.; Kuhl, M. A new system for threedimensional tracking of motile microorganisms. Appl. Environ. Microbiol. 2000, 66, 2238-2242.

(15) Oldenburg, S. J.; Averitt, R. D.; Westcott, S. L.; Halas, N. J. Nanoengineering of optical resonances. Chem. Phys. Lett. 1998, 288, 243-247.

(16) Knight, M. W.; Wu, Y.; Lassiter, J. B.; Nordlander, P.; Halas, N. J. Substrates Matter: Influence of an Adjacent Dielectric on an Individual Plasmonic Nanoparticle. Nano Lett. 2009, 9, 2188-2192.

(17) Wu, Y. P.; Nordlander, P. Finite-Difference Time-Domain Modeling of the Optical Properties of Nanoparticles near Dielectric Substrates. J. Phys. Chem. C 2010, 114, 7302-7307.

(18) Zhang, S. P.; Bao, K.; Halas, N. J.; Xu, H. X.; Nordlander, P. Substrate-Induced Fano Resonances of a Plasmonic: Nanocube: A Route to Increased-Sensitivity Localized Surface Plasmon Resonance Sensors Revealed. Nano Lett. 2011, 11, 1657-1663.

(19) Hentschel, M.; et al. Transition from Isolated to Collective Modes in Plasmonic Oligomers. Nano Lett. 2010, 10, 2721-2726.

(20) Lassiter, J. B.; et al. Fano Resonances in Plasmonic Nanoclusters: Geometrical and Chemical Tunability. Nano Lett. 2010, 10, 3184-3189.

(21) Fan, J. A.; et al. Fano-like Interference in Self-Assembled Plasmonic Ouadrumer Clusters. Nano Lett. 2010, 10, 4680-4685.

(22) Wu, C.; et al. Fano-resonant asymmetric metamaterials for ultrasensitive spectroscopy and identification of molecular monolayers. Nat. Mater. 2012, 11, 69-75.

(23) Giannini, V.; Francescato, Y.; Amrania, H.; Phillips, C. C.; Maier, S. A. Fano Resonances in Nanoscale Plasmonic Systems: A Parameter-Free Modeling Approach. Nano Lett. 2011, 11, 2835-2840.

(24) Gallinet, B.; Martin, O. J. F. Influence of Electromagnetic Interactions on the Line Shape of Plasmonic Fano Resonances. ACS Nano 2011, 5, 8999-9008.

(25) Pryce, I. M.; Aydin, K.; Kelaita, Y. A.; Briggs, R. M.; Atwater, H. A. Highly Strained Compliant Optical Metamaterials with Large Frequency Tunability. Nano Lett. 2010, 10, 4222-4227.

(26) Kottmann, J. P.; Martin, O. J. F. Retardation-induced plasmon resonances in coupled nanoparticles. Opt. Lett. 2001, 26, 1096-1098.

(27) Hao, F.; Larsson, E. M.; Ali, T. A.; Sutherland, D. S.; Nordlander, P. Shedding light on dark plasmons in gold nanorings. Chem. Phys. Lett. 2008, 458, 262-266.

(28) Kats, M. A.; Yu, N. F.; Genevet, P.; Gaburro, Z.; Capasso, F. Effect of radiation damping on the spectral response of plasmonic components. Opt. Express 2011, 19, 21748-21753.

(29) Alzar, C. L. G.; Martinez, M. A. G.; Nussenzveig, P. Classical analog of electromagnetically induced transparency. Am. J. Phys. 2002, 70, 37-41.

(30) Lassiter, J. B.; et al. Designing and Deconstructing the Fano Lineshape in Plasmonic Nanoclusters. Nano Lett. 2011, 12, 10581062. 